\title{
On the Surface Sensitivity of Angular Scans in LEIS
}

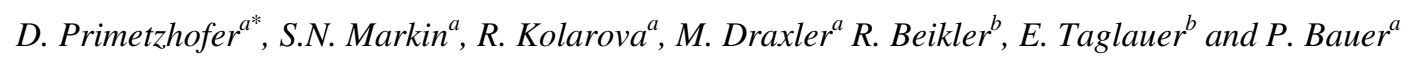

${ }^{a}$ Institut für Experimentalphysik, Johannes Kepler Universität Linz, A-4040 Linz, Austria.

${ }^{b}$ Max-Planck-Institut für Plasmaphysik, EURATOM Association, D-85748 Garching bei München, Germany.

\begin{abstract}
$\mathrm{A} \mathrm{Cu}(100)$ surface has been investigated by means of time-of-flight low-energy ion scattering using ${ }^{4} \mathrm{He}^{+}$ions. Polar and azimuth scans have been performed. The evaluation of the obtained spectra demonstrates the influence of background contributions to the overall yield. Selection of single scattering processes was achieved by background subtraction. This allows for high surface sensitivity even for neutral scattering spectra.
\end{abstract}

\section{PACS numbers:}

34.50.Dy, 68.49.Sf, 79.20.Rf

\section{Keywords:}

Low energy ion scattering, single crystal, background correction, $\mathrm{Cu}(100)$

\section{Introduction}

In low-energy ion scattering (LEIS) usually noble gas ions are used to investigate the surface of a (solid) sample. Ions and neutrals that are scattered by a large angle are detected [1 ]. In such experiments, the information depth is limited to a few atomic layers [2]. In the LEIS regime (energy range $1-10 \mathrm{keV}$ ) the scattering potential is given by a screened Coulomb potential

$$
\mathrm{V}(\mathrm{r})=\frac{\mathrm{Z}_{1} \mathrm{Z}_{2} \mathrm{e}^{2}}{\mathrm{r}} \cdot \Phi\left(\frac{\mathrm{r}}{\mathrm{a}}\right),
$$

with $\mathrm{Z}_{1}$ and $\mathrm{Z}_{2}$ the atomic numbers of ion and scattering centre, respectively, and $\Phi(\mathrm{r} / \mathrm{a})$ the screening function with the characteristic screening length a [3],[4]. Usually, this screening length is corrected by a factor $\mathrm{c}_{\mathrm{a}}$ in order to match simulation and experiment.

A common way to deduce information on the interaction potential is to record the yield of backscattered particles when changing the angular orientation of the sample: (i) in a polar scan the angle of incidence $\alpha$ (measured with respect to the surface normal) and (ii) in the azimuth scan the angle $\varphi$ is varied. These experimental scans can be compared to computer simulations obtained from different codes (e.g., FAN [5], MARLOWE [6]). By changing the input parameters in the 
simulation, e.g. screening length correction factor or inelastic energy loss, the calculated results can be fitted to the experimental data, thereby providing information on the scattering process.

However, also the experimental information, to which the simulations are compared, depends on evaluation details: an important question is for instance, how the experimental yield is defined. This is of tremendous importance since in LEIS not only the observed intensity but also the shape of the spectrum exhibits a strong dependence on the angles $\alpha$ and $\varphi$. Especially the background arising from multiple scattering and contributions from deeper layers has a strong influence on the resulting yield. Therefore, the way how the backscattered yield is deduced from experimental spectra influences the information obtained.

\section{Experiment}

The experiments were performed using the TimeOf-Flight- (TOF-) LEIS setup ACOLISSA [7] with a scattering angle $\theta$ of $129^{\circ}$ and a detector acceptance angle of $0.92^{\circ}$. The angular precision of the manipulator is $\pm 0.1^{\circ}$ and $\pm 0.2^{\circ}$ for polar and azimuth scans, respectively. The time resolution of the system is set to typically 10 to $25 \mathrm{~ns}$. A post acceleration voltage can be applied along part of the flight path between sample and detector to separate backscattered ions from neutrals. The primary beam current is set to 25 to $100 \mathrm{nA}$ in full beam mode, yielding 5 to $20 \mathrm{pA}$ in the chopped beam mode. The beam current remains constant within $10 \%$ after thermal equilibration $(\sim 2 \mathrm{~h})$. At normal incidence, the beam spot on the sample was found to be smaller than $1 \mathrm{~mm}$ in diameter. From this the safe range of incident angles follows $(\alpha<$ $65^{\circ}$ ) ensuring that the whole irradiated spot is visible for the detector.

The measurements were performed using a $\mathrm{Cu}(100)$ single crystal surface. ${ }^{4} \mathrm{He}^{+}$ions with energies in the range of $1.8-8 \mathrm{keV}$ were used as projectiles. Azimuth scans $\mathrm{Y}(\varphi)$ and polar scans $\mathrm{Y}(\alpha)$ were measured in an angular range of $140^{\circ}$ and $100^{\circ}$, respectively.

The sample was prepared by repetitive sputtering annealing cycles, performed with $3 \mathrm{keV} \mathrm{Ar}^{+}$ions and subsequent heating to $\sim 650 \mathrm{~K}$. The purity of the sample and the crystal structure were checked by Auger electron spectroscopy (AES) and lowenergy electron diffraction (LEED), respectively.

\section{Results und Discussions}

In TOF-LEIS spectra in a wide TOF range (typically $2 \mu \mathrm{s}$ ) are obtained. When a single crystal is used as a target, the spectra exhibit features with strong angular dependence, due to the high degree of symmetry of the system.

At normal incidence, azimuth scans provide high surface sensitivity, due to advantageous combination of channeling and angular dependent blocking. Thus, projectiles can be scattered into the detector in a single collision only by atoms in the outermost one or two atomic layers. However, the acquired spectra will contain contributions due to backscattering from deeper layer atoms in multiple collisions. This decreases surface sensitivity. Nevertheless, the intensity ratio of one vs. two visible atomic layers should be observable in the azimuth scans if one manages to extract the correct surface scattering contributions from the obtained spectra.

In Fig. 1, a typical spectrum is shown for $3 \mathrm{keV}$ $\mathrm{He}^{+}$projectiles and a $\mathrm{Cu}(100)$ surface acquired in double alignment geometry with post acceleration voltage applied. Therefore, the contributions of (positive) ions and neutrals are separated in the spectrum, leading to the following features:

$$
\begin{array}{ll}
\text { ion peak: } & \text { detected ions } \\
\text { surface peak: } & \text { detected }
\end{array}
$$
neutrals backscattered in the outermost layers 
(iii)

background: multiple

scattering and deeper layer contributions

Without applying post acceleration scattered ions would end up in the surface peak since no azimuth dependence of the ion yield for $3 \mathrm{keV} \mathrm{He}^{+}$was observed. This indicates that the detected ions are almost solely backscattered by the first atomic layer in a single collision, and contributions due to reionization are negligible [8].

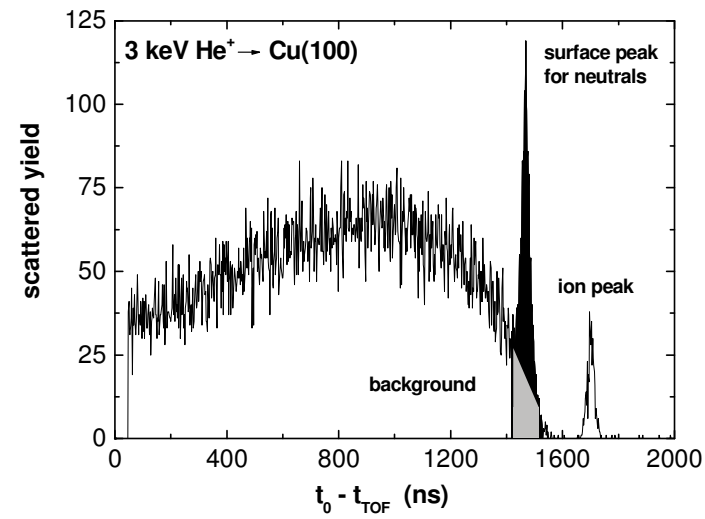

Fig. 1: Typical TOF-LEIS spectrum for $3 \mathrm{keV} \mathrm{He}^{+}$ions and $\mathrm{Cu}(100)$ obtained in double alignment geometry: $\alpha=0, \phi=0$, as a function of $\mathrm{t}_{0}-\mathrm{t}_{\mathrm{TOF}}$, with the time delay $\mathrm{t}_{0}=6.45 \mu \mathrm{s}$. The grey shaded area indicates the assumed background at the position of the surface peak.

For evaluation different parts of the spectrum can be taken into account. The use of different TOF windows is equivalent to restricting the final energy of the detected backscattered particles to different values. This limits the maximum path length in the target because of inelastic energy losses due to electronic stopping along the trajectory [9]. Of course, due to kinematical losses the number of possible collisions by specific angles depends on the selected TOF window. Consequently, the setting of this TOF window corresponds to a specific selection of trajectories.

The following types of evaluation are possible:

full TOF window: integration of the full TOF window is equivalent to accepting all types of trajectories including multiple scattering and contributions from deeper layers.

small TOF window: integration of a small TOF window around the surface peak is equivalent to restricting the possible trajectories (path length), but still permitting multiple scattering processes to contribute.

(iii) small TOF window with background subtraction: integration of a small TOF window around the surface peak and subtraction of an assumed background is equivalent to selecting single scattering processes in the two uppermost layers for the investigated system.

The choice of the integration limits will change the resulting information significantly. Subtracting background contributions will lead to most instructive information. In this case, the width of the blocking minima and the intensity ratio between maxima (random exit) and minima (double alignment) will not be blurred by projectiles that have penetrated below the surface layers. 

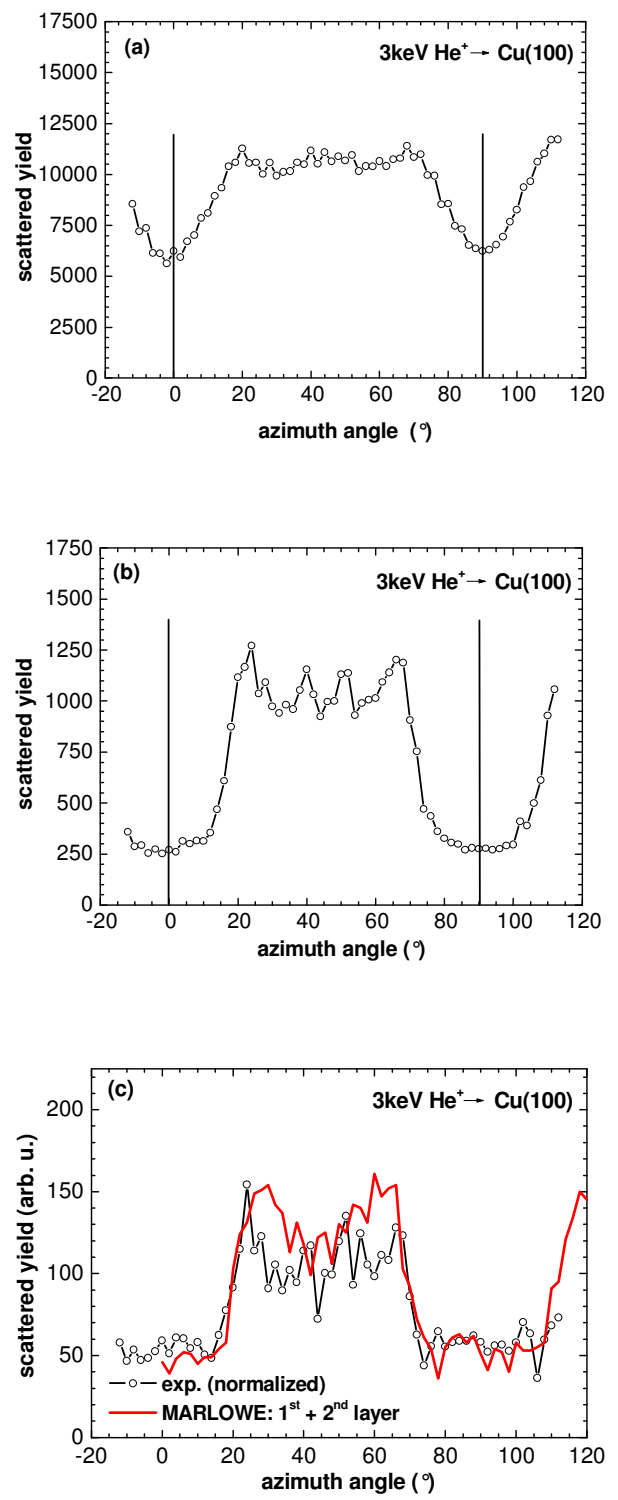

Fig. 2: (a) Experimental azimuth scan obtained by integrating the full available TOF window. (b) Experimental azimuth scan obtained by integrating a time window of the full width of the surface peak centered at the peak maximum. (c) Experimental azimuth scan with TOF window like in (b) but without background. The solid line shows the results of a MARLOWE simulation for the two uppermost ML. All scans and simulations: $3 \mathrm{keV} \mathrm{He}{ }^{+}$ions and $\mathrm{Cu}(100)$.

Fig. 2a shows an azimuth scan for $3 \mathrm{keV} \mathrm{He}^{+}$ions integrating the full TOF window (case (i)). The spectrum shows the expected fourfold symmetry indicating good crystal quality. The minima correspond to double alignment. The half-width of the minima is about $11^{\circ}$. The maximum exhibits a broad plateau with some internal structure. Note the absence of a plateau in the minima which makes it difficult to interpret the intensity ratio maximum $\left(\mathrm{I}_{\max }\right)$ to minimum $\left(\mathrm{I}_{\min }\right)$. Fig. $2 \mathrm{~b}$ presents the results obtained for a TOF window restricted to the full width of the surface peak for the identical dataset. There are obvious differences concerning the yield, the structure in the maximum and the shape of the minimum, yielding an intensity ratio $\mathrm{I}_{\max } / \mathrm{I}_{\min }=\sim 3.5$. The half-width of the minima is determined to $18^{\circ}$. Finally, the scan presented in Fig. 2c is obtained for the same TOF window as in Fig. $2 b$ by subtracting a linear background. The subtraction and integration was performed using the computer program LabView [ 10 ] and checked using standard evaluation routines. The background subtraction results in a decrease in intensity and a further increase of the half-width of the minima which is $21^{\circ}$. The structures at $40^{\circ}$ and $50^{\circ}$ which have been observed in Fig. 2b are not visible anymore. Note that due to the lower yield the statistical spread is larger. The intensity ratio $I_{\max } / I_{\min } \cong 2$ is as expected for contributions from two or one atomic layers to the yield.

These results support the importance of proper background subtraction. The data shown in Fig. $2 \mathrm{a}$ indicate a strong azimuth dependence of the background. As visible in Fig. 1 the background to the left of the surface peak (low-energy background, LEB) is obviously higher than to the right (high-energy background, HEB). Since one should expect a smooth and continuous transition from LEB to HEB, the background subtraction was performed accordingly (see Fig. 1).

For a further evaluation of our results, layer resolved MARLOWE computer simulations have been performed. These simulations corroborate the information obtained for the different evaluation procedures. In Fig. 3 the calculated intensity is presented as a function of the azimuth angle for the first 5 layers of $\mathrm{Cu}(100)$ for the experimental 
conditions. As expected, the intensity of the first layer does not show any angular dependence. The contribution from the second layer is dominated by the effects of blocking and focusing. This focusing causes an enhancement of detected intensity when the second layer atoms become visible for the detector. The simulation indicates a strong contribution of deeper layers to the total intensity. In the simulation, a crystal size of $18 \mathrm{ML}$ was used. By summing up the contributions of individual layers it was found that $50 \%$ of the total backscattered intensity is due to projectiles backscattered from layers 6 to 18 . Additionally, the contributions of individual layers demonstrate a different azimuth dependence concerning intensity and width of the minima. This leads to the conclusion that the number of layers chosen in a simulation will strongly influence the shape of the resulting angular scans.

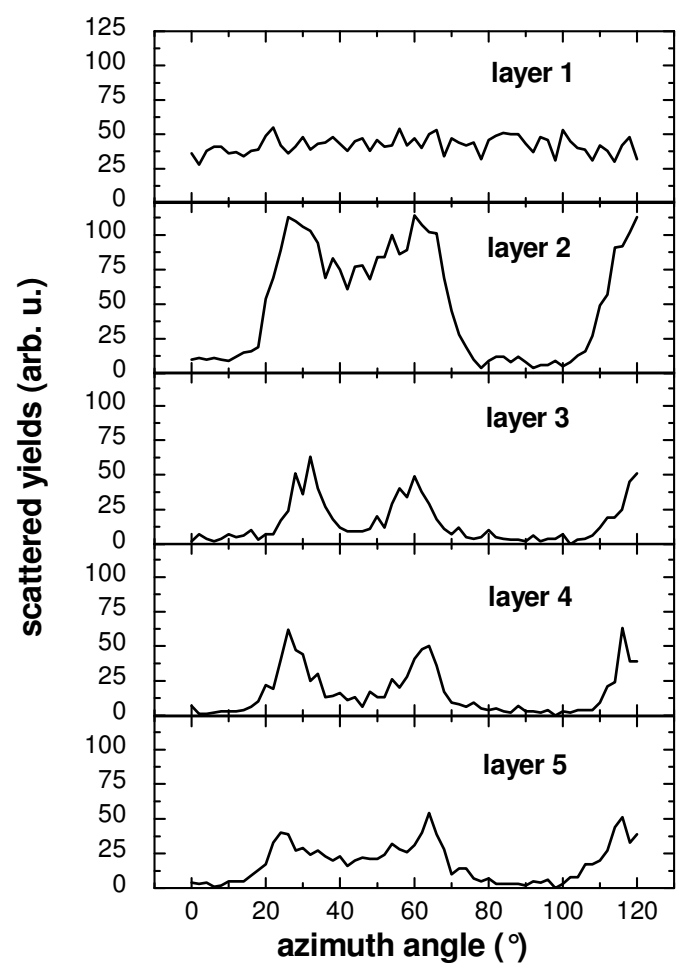

Fig. 3: Layer resolved MARLOWE simulation for $3 \mathrm{keV} \mathrm{He}^{+}$ and $\mathrm{Cu}(100)$.

In Fig. 2c the azimuth dependence of the surface peak area after background subtraction is compared to MARLOWE results for the first two ML. This yields a good agreement in the intensity ratio $I_{\max } / I_{\min }$. A possible background contribution and its subtraction should therefore always be considered in evaluation of TOF-LEIS spectra. Note that the structure in the maximum obtained by the simulation is more pronounced than in the experiment. Further improvement is still possible by optimization of simulation parameters like screening length correction, atomic vibrations and inelastic losses.

\section{Conclusions}

The aim of the experiments described above was to show the qualitative and quantitative influence of the background due to multiple scattering and deeper layer contributions in TOF-LEIS spectra evaluation. For this purpose a $\mathrm{Cu}(100)$ surface was investigated with low-energy $\mathrm{He}^{+}$ion scattering. Azimuth scans in channeling conditions have been recorded and evaluated using different energy windows and background subtraction. This led to changes in the intensities and the angular widths of the structures. Layer resolved MARLOWE computer simulations have been performed to obtain information on the origin of the backscattered intensities. By comparing these simulations to the experimental results it was found that background subtraction is important in order to select scattering processes which occur only in the surface.

\section{Acknowledgements}

This work was partly supported by the Austrian Science Fund (FWF) under contract number P16469-N08. 


\section{References}

[1] H. Niehus, W. Heiland and E. Taglauer, Surf. Sci. Rep. 17 (1993) 213.

[2] Th. Fauster, Vacuum 38 (1988) 129.

[3] M.A. Karolewski, Nucl. Instr. Meth. B 243 (2006) 43.

[4] A. Schüller, S. Wethekam, A. Mertens, K. Maass, H. Winter, K. Gärtner, Nucl. Instr. Meth. B 230 (2005) 172.

[5] H. Niehus, R. Spitzl, Surf. Interface Anal. 17 (1991) 287.

[6] M.T. Robinson, I.M. Torrens, Phys. Rev. B 9 (1974) 5008.

[7] M. Draxler, S.N. Markin, S.N. Ermolov, K. Schmid, C. Hesch, R. Gruber, A. Poschacher, M. Bergsmann, P. Bauer, Vacuum 73 (2004) 39.

[8] M. Draxler, R. Gruber, H. H. Brongersma, P. Bauer, Phys.

Rev. Lett. 89 (2002) 263201.

[9] M. Draxler, R. Beikler, E. Taglauer, K. Schmid, R. Gruber, S.

N. Ermolov, P. Bauer, Phys. Rev. A 68 (2003) 022901.

[10] LabView 6.0 @ 2000 National Instruments. 